Annuaire suisse de politique de développement

16 | 1997

Environnement et développement, 5 ans après Rio

\title{
4. Culture et science
}

\section{(2) OpenEdition}

1 Journals

Édition électronique

URL : http://journals.openedition.org/aspd/790

DOI : 10.4000/aspd.790

ISSN : 1663-9669

Éditeur

Institut de hautes études internationales et du développement

\section{Édition imprimée}

Date de publication : 1 mars 1997

Pagination : 113-117

ISSN : 1660-5934

\section{Référence électronique}

"4. Culture et science», Annuaire suisse de politique de développement [En ligne], 16 | 1997, mis en ligne le 07 août 2012, consulté le 08 septembre 2020. URL : http://journals.openedition.org/aspd/790 DOI : https://doi.org/10.4000/aspd.790 


\section{CULTURE ET SCIENCE}

\section{RECHERCHE ET COOPÉRATION SCIENTIFIQUE}

\section{$\square$ Conférence internationale sur le partenariat scientifique}

La première conférence internationale «Scientific Research Partnership for Sustainable Development - North-South and South-South Dimensions » s'est tenue du 5 au 7 mars 1996 à l'Université de Berne. Présidée par le professeur Thierry Freyvogel, la rencontre a été mise sur pied par la KFPE ${ }^{1}$ avec le concours de la DDC, de l'IUED, du Fonds national pour la recherche scientifique (PPEModule 7) et de l'ASSN (Académie suisse des sciences naturelles). Elle a connu un succès remarquable : 370 participants de 50 pays du Sud (représentant un quart des participants) et 17 pays industrialisés ont discuté des moyens d'associer leurs recherches. Le spectre des disciplines représentées était très large, des ingénieurs agronomes aux historiens, sans oublier les représentants des sciences sociales et de l'économie.

Les pays de l'OCDE comptent 3500 scientifiques et ingénieurs pour un million d'habitants. Ce chiffre tombe à 400 universitaires par million d'habitants en Amérique latine, à 200 en Asie et à une centaine en Afrique. Les nations industrialisées investissent dans la recherche une part dix fois plus importante de leur produit national brut que les pays en développement. Ces chiffres révèlent les profondes disparités entre le Nord et le Sud sur le plan du savoir comme sur celui du niveau de vie. La volonté d'instaurer un développement durable et de résoudre les problèmes écologiques globaux présente un défi énorme et suppose également qu'on élimine le déséquilibre des potentiels de recherche. C'est dans cet esprit que s'est déroulée la Conférence, qui s'est donné trois objectifs :

口 d'attirer l'attention de la communauté scientifique et des décideurs sur l'intérêt de la recherche pour le développement et de démontrer l'importance du partenariat avec et entre les pays du Sud ;

- de passer en revue les programmes de recherches conjoints déjà en cours, mettant en évidence les bénéfices d'un partenariat ;

a de discuter des mesures à prendre pour accroître les capacités de recherches dans les pays en développement et de définir des types de collaboration entre des partenaires différents.

La discussion fut lancée par l'analyse de la stratégie suisse pour la promotion de la recherche dans les pays en développement et elle a abouti par la définition en définissant des types de collaboration entre partenaires travaillant dans des conditions très différentes.

1 Instituée en 1994 pour une période de trois ans la KFPE (Commission suisse pour le partenariat scientifique avec les pays en développement) est une commission de la Conférence des académies scientifiques suisses (CASS). Une trentaine de personnalités suisses en font partie, provenant du monde académique, de l'économie, du commerce extérieur, de la politique, des ONG, du Fonds national de la recherche scientifique, de la DDC, d'offices fédéraux et d'institutions suisses de recherches spécialisées. Elle a pour but d'aménager et de consolider les capacités de recherche dans les pays du Sud, en favorisant un partenariat scientifique orienté vers une politique de développement à long terme. Secrétariat KFPE, Bärenplatz 2, 3011 Berne, tél. 031/311.06.01. 
Une publication ${ }^{2}$ résume les travaux de la conférence et met en évidence un certain nombre de conclusions. Les moyens pour combler les lacunes en matière de recherche se sont exprimés lors des tables rondes, ne laissant pas apparaître une véritable stratégie commune, mais plutôt une série de propositions. Un consensus s'est pourtant dégagé sur le principe d'un partenariat, mais qui doit s'éloigner de l'état d'esprit « donateur-bénéficiaire ». L'importance de la valeur du savoir indigène et de la culture traditionnelle, ainsi que la nécessité d'un partage des connaissances ont été relevées. Les potentiels et les limites d'un partenariat dans la recherche ont également fait l'objet d'une discussion. Le potentiel de collaboration internationale dans la recherche pour le développement est immense, mais les obstacles pour une mise en œuvre équitable restent considérables. La tendance à considérer la science du Nord comme une science internationale, alors que la contribution du Sud est considérée comme locale, reflète un des plus grands obstacles pour une collaboration authentique. La dominance du Nord peut affecter de nombreux aspects de cette collaboration; du choix des partenaires au planning de recherches, sans oublier la gestion des fonds, l'accès à l'information, les possibilités de publications de même que l'utilisation des résultats obtenus et les droits d'auteurs. Un des moyens évoqués pour éviter un tel déséquilibre est de renforcer la collaboration Sud-Sud, ce qui ne doit pas être perçu comme le remplacement d'un partenariat Nord-Sud, mais s'ajouter à celui-ci.

La stratégie élaborée par la Suisse en 1993 pour promouvoir la recherche dans les pays en développement sera revue à la lumière des nouveaux éléments abordés lors de cette conférence.

1994 Recherche et coopération scientifique, pp. 107-109.

\section{$\square$ Programme national de recherche $n^{\circ} 42$}

En 1993, le Conseil fédéral a chargé le Fonds national suisse de la recherche scientifique (FNRS) de réaliser le programme national de recherche (PNR) 42 « Fondements et possibilités de la politique extérieure suisse » ${ }^{3}$.

Les problèmes actuels de la politique extérieure suisse soulèvent de nombreuses questions qui nécessitent une clarification scientifique. Le PNR 42 vise à élaborer les fondements de la politique extérieure sur un plan scientifique, identifier les marges de manœuvre possibles, fournir des bases de décision et définir de nouvelles stratégies. Officiellement lancé en octobre 1996, il est doté d'un crédit de 16 millions de francs et les recherches seront terminées d'ici à la fin 1999. Après une phase d'évaluation 47 projets ont été acceptés sur un ensemble de 193 propositions. ${ }^{4}$ Les relations Suisse-Tiers Monde feront l'objet d'une dizaines de projets, principalement pour des sujets économiques (commerce, investissements privés) ou du rôle de la Suisse dans des organisations internationales (ONU, OIT, $\mathrm{OMC}$ ).

${ }^{2}$ Research Partnerships for Common Concerns, Proceedings of the International Conference on Scientific Research Partnership for Sustainable Devopment - North-South and South-South Dimensions, D. Maselli \& B. Sottas (eds.), LIT Verlag, 1996.

3 Depuis 1975, les PNR servent de base scientifique à la politique du Conseil fédéral. Ils doivent contribuer à résoudre des problèmes urgents et d'intérêt national sur le plan scientifique, politique et social. Le FNRS est chargé de leur exécution.

4 Les 47 projets, ainsi que leurs responsables, sont présentés dans une brochure « Fondements et possibilités de la politique extérieure suisse », PNR 42, Politique extérieure suisse. Disponible au secrétariat du FNRS, Wildhainweg 20 , 3001 Berne, tél. 031/308.22.22. 


\section{Tableau $\mathrm{n}^{\mathrm{0}} \mathbf{1 7}$ \\ Etat d'avancement de divers PNR qui abordent des question liées à la coopération au développement ou aux relations Nord-Sud en 1996}

PNR 28

Thèmes

Economie extérieure et politique de développement
Objectif

Le programme a pour but de développer des stratégies d'adaptation face aux changements internationaux attendus. Il contient d'une part le domaine des relations économiques extérieures et d'autre part celui de la politique du développement. Il analysera les différents développements dus aux relations économiques extérieures, tentera de mesurer l'efficacité des instruments de politique extérieure et fera des propositions quant à l'implantation de nouvelles mesures. Une approche globale essaiera de clarifier les interdépendances multiples entre économie extérieure et politique de développement. Le programme devra présenter des résultats qui soient convertibles en propositions et stratégies d'action cohérentes pour les acteurs publics et privés.

\author{
Nombre de projets \\ 28 \\ Budget \\ 5 millions de francs \\ Remarques
}

Publication du rapport de synthèse 20 du PNR 28, « Wirtschaftsverfassungartikel für die Schweiz », Disponible en français et en allemand, à l'adresse suivante : Programmleitung NFP 28, Birkenweg 7, 8890 Einsiedeln, tél/fax. 055/412.34.89.

PNR 31
Thèmes
Changements climatiques et
catastrophes naturelles

Objectif

Les problèmes propres aux changements climatiques et aux interactions " climat-écosystèmescatastrophes naturelles-société » constituent le fil conducteur du programme. Nous devons améliorer notre compréhension du comportement de l'environnement lors d'événements météorologiques à court terme ou lors de changements climatiques à long terme. Les aspects économiques, politiques, administratifs et sociologiques devront être pris en considération.
PNR 39

\section{Thèmes}

Migrations et relations interculturelles

\begin{abstract}
Objectif
Le programme a pour but d'éclairer la complexité du phénomène des migrations et des relations interculturelles. L'accent sera mis sur la dynamique des processus de migrations, sur leurs conséquences sociales, économiques et politiques, ainsi que sur les possibilités politiques d'influencer ces processus aux échelons national et international. On attend du programme des bases de décision pour les autorités politiques, une aide pratique aux personnes engagées dans l'assistance aux migrants, ainsi qu'une contribution à dépassionner le débat sur les migrations.
\end{abstract}

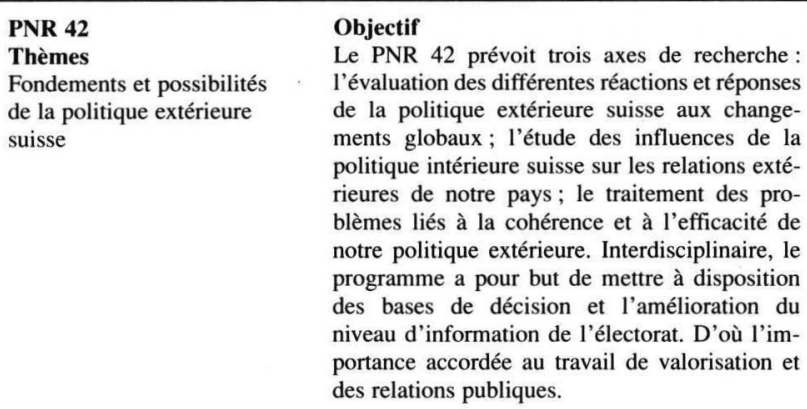

\section{Nombre de projets}

58

\section{Budget}

20 millions de francs

Remarques

Les travaux de recherche seront probablement terminés en 1997.

\section{Nombre de projets}

25

\section{Budget}

8 millions de francs

\section{Remarques}

Suite à une mise au concours, le Fonds national a reçu 123 esquisses de projets. Au courant de l'année 1996, environ 25 projets de recherche ont débuté.

\section{Nombre de projets \\ 47 \\ Budget}

16 millions de francs

Remarques

Officiellement lancé en octobre 1996, il sera en cours jusqu'à fin 1999. 
L'objet du PNR 42 est l'ensemble du domaine des relations extérieures dans une perspective aussi bien actuelle qu'historique. L'éventail des thèmes acceptés va des thèmes classiques de la politique extérieure (relations avec les autres Etats, organisations internationales, etc.) à leurs aspects plus particuliers (politique économique extérieure, coopération au développement, politique de sécurité, entre autres). Ils sont regroupés en trois axes principaux : le changement global et la politique extérieure suisse, la dimension interne des relations extérieures de la Suisse et enfin la cohérence et l'efficacité de la politique extérieure suisse.

\section{SOURCES}

Research Partnerships for Common Concerns, Proceedings of the International Conference on Scientific Research Partnership for Sustainable Development - North-South and South-South Dimensions, D. Maselli \& B. Sottas (eds.), LIT Verlag, 1996. (Actes du séminaire)

$E+D, \mathrm{n}^{\circ} 49$, juillet 1996 « Nord et Sud associent leurs recherches ».

Horizon, mars 1996 « Repenser la recherche Nord-Sud ».

Journal de Genève, 6 mars 1996 « Isolée en Europe, la recherche suisse collabore avec le Sud ».

Journal de Genève, 5 octobre 1996 « Lancement du programme de recherche sur la politique extérieure ».

La Liberté, 8 mars 1996 « Le partenariat se relève une chose délicate ».

Neue Zürcher Zeitung, 5. März 1996 « Okologie - Forschung über ein Weltproblem », 7. März 1996 « Forschung für eine gemeinsame Entwicklung », 5. Oktober 1996 «Schweizer Aussenpolitik als Forschungsobjekt ».

Tages-Anzeiger, 4. März 1996 "Forschende auf Südsicht lenken », 12. März 1996 " Forschung im Süden in grosser Not ».

Fondements et possibilités de la politique extérieure suisse, PNR 42, Politique extérieure suisse, FNRS, septembre 1996. Les revues Agenda parues en 1996, FNRS.

\section{RÉGLEMENTATION DU COMMERCE DE L'ART EN SUISSE}

Répondant aux appels de plus en plus pressants de l'étranger et suite à plusieurs affaires survenues en Suisse, l'Office fédéral de la culture a constitué le 7 janvier 1990 un groupe de travail chargé d'étudier la question de l'exportation de biens culturels suisses et du commerce de biens culturels étrangers en Suisse. Il devait également formuler des propositions de mesures à mettre en œuvre. Le groupe de travail a remis son rapport' à l'Office fédéral de la culture en janvier 1991. Le rapport proposait principalement deux mesures :

- la ratification de la Convention de l'UNESCO de 1970 concernant les mesures à prendre pour interdire et empêcher l'importation, l'exportation et le transfert de propriété illicite des biens culturels ;

- la création d'une disposition constitutionnelle donnant à la Confédération compétence pour réglementer l'importation et l'exportation de biens culturels.

Le rapport est rendu public plus d'une année après sa parution en avril 1992. Le même mois, la Déclaration de Berne présentait à la presse une nouvelle publication "Götter, Gräber und Geschäfte, Von der Plünderung fremder Kulturen », qui soulignait notamment le rôle de plaque tournante de la Suisse dans le commerce de l'art.

\footnotetext{
Rapport du groupe de travail à l'intention de l'Office fédéral de la culture et du Département fédéral de l'intérieur, Le commerce, l' exportation et l'importation de biens culturels en Suisse. Portée nationale et internationale ; problèmes, solutions et effets, janvier 1991.
} 
Parallèlement, une nouvelle Convention internationale (Convention d'Unidroit du 24 juin 1995 sur les biens culturels volés ou illicitement exportés) était négociée à Rome. La Suisse s'est passablement impliquée dans la rédaction de ce texte, notamment par la présidence du comité d'experts de Pierre Lalive d'Epinay, professeur de droit à l'Université de Genève.

Le Conseil fédéral a lancé, en deux temps, une double procédure de consultation. La première consultation a été ouverte en septembre 1993 pour la ratification de la Convention de l'UNESCO de 1970 et l'introduction d'un article constitutionnel habilitant la Confédération à légiférer sur l'importation, l'exportation et le commerce des biens culturels. En janvier 1996, le Conseil fédéral lançait la seconde consultation se rapportant à la Convention d'Unidroit et la possibilité de sa signature par la Suisse.

Les résultats des deux procédures de consultation ont été rendus publics en 1996. Une écrasante majorité des milieux concernés souhaite donner à la Confédération la compétence pour légiférer dans le domaine du commerce international de biens culturels, approuve la ratification de la Convention de l'Unesco et celle d'Unidroit et est favorable à l'élaboration d'une loi fédérale.

Suite aux résultats de cette double consultation, le Conseil fédéral a pris deux mesures, d'une part, la Convention d'Unidroit a été signée par la Suisse en juin 1996 à Rome, d'autre part un groupe de travail interdépartemental sous la conduite du DFI a été institué afin de coordonner la suite des travaux.

Département fédéral de l'intérieur (Office fédéral de la culture), Communiqués de presse de janvier et juin 1996, consacrés aux résultats des procédures de consultation.

1997 Commerce, importation et exportation de biens culturels : états de la réglementation en Suisse, p 271. 